\title{
Near-Infrared Light and Upconversion Nanoparticle Defined Nitric Oxide-Based Osteoporosis Targeting Therapy
}

Jing Ye $e^{a, b, c \neq}$, Junkai Jiang ${ }^{b}$, Zhirui Zhou ${ }^{b}$, Zhenzhen Weng ${ }^{b, c}$, Yingying Xu ${ }^{b, c}$, Lubing Liu ${ }^{b, c}$, Wei Zhang $^{b, c}$, Yifei Yang ${ }^{d}$, Jun Luo ${ }^{a *}$, and Xiaolei Wang ${ }^{b, c *}$

a. The Department of Rehabilitation Medicine, the Second Affiliated Hospital of Nanchang University, Nanchang University, Jiangxi, 330006 (P.R. China)

b. The National Engineering Research Center for Bioengineering Drugs and the Technologies, Institute of Translational Medicine, Nanchang University, Nanchang, Jiangxi, 330088 (P.R. China)

c. College of Chemistry, Nanchang University, Nanchang, Jiangxi, 330088 (P.R. China)

d. School of Public Health \& Jiangxi Provincial Key Laboratory of Preventive Medicine, Nanchang, Jiangxi, 330088 (P.R. China) 


\section{Figures}

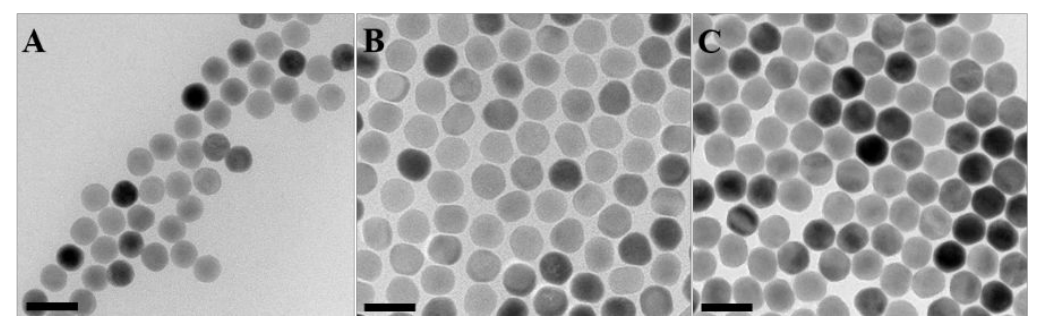

Figure S1. A-C) TEM images of $\mathrm{NaYF}_{4}: \mathrm{Yb} / \mathrm{Tm}(25 / 0.5 \%)$ core nanocrystals, $\mathrm{NaYF}_{4}: \mathrm{Yb} / \mathrm{Tm}$ $(25 / 0.5 \%)$ core/ $\mathrm{NaYF}_{4}: \mathrm{Nd} / \mathrm{Yb} \quad(20 / 10 \%)$ shell nanocrystals and $\mathrm{NaYF}_{4}: \mathrm{Yb} / \mathrm{Tm} \quad(25 / 0.5 \%)$ core $/ \mathrm{NaYF}_{4}: \mathrm{Nd} / \mathrm{Yb}(20 / 10 \%)$ shell/ $\mathrm{NaYF}_{4}$ shell nanocrystals, respectively. Scale bar: $50 \mathrm{~nm}$.

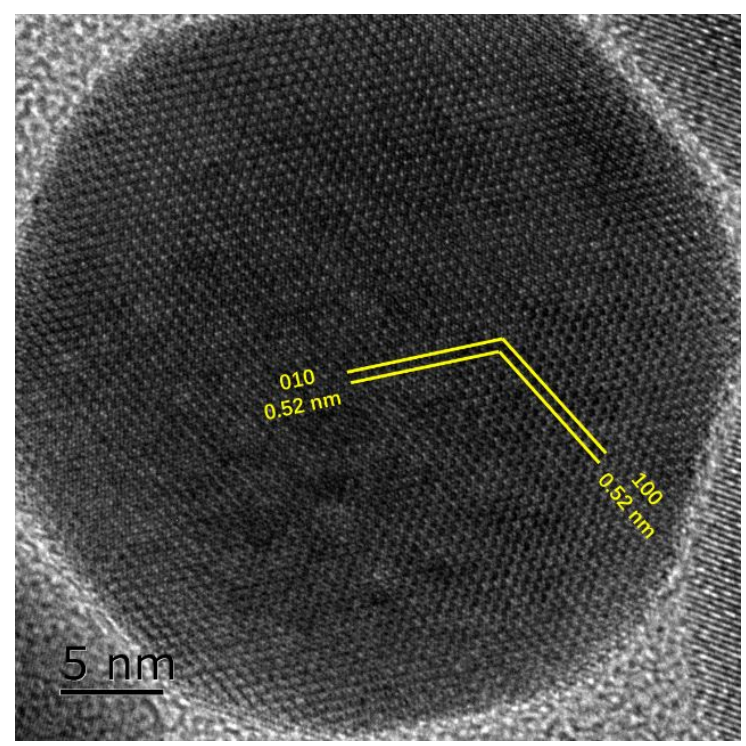

Figure S2. HRTEM image of the $\mathrm{NaYF}_{4}: \mathrm{Yb} / \mathrm{Tm}$. The HRTEM image of UCNPs showed that the lattice distance was $0.52 \mathrm{~nm}$, corresponding to the typical plane (110 and 010) of the hexagonal $\mathrm{NaYF}_{4}$ structure. 


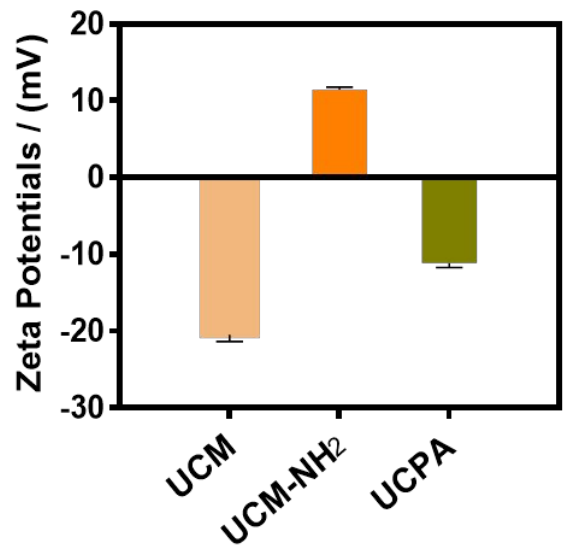

Figure S3. Zeta potentials of UCM, UCM-NH ${ }_{2}$ and UCPA. Data are means \pm s.d. $(n=3)$.
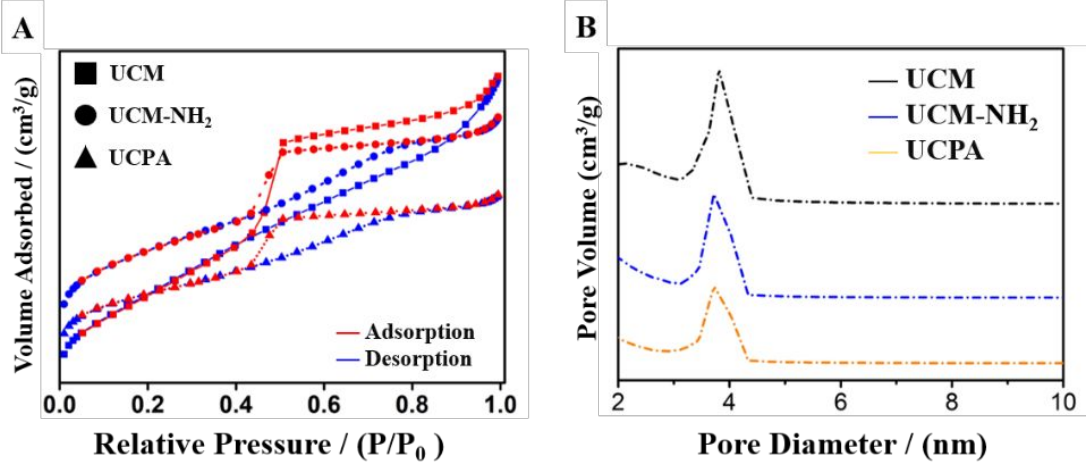

Figure S4. A) $\mathrm{N}_{2}$ adsorption-desorption isotherms and B) pore-size distribution of UCM, UCM-

$\mathrm{NH}_{2}$ and UCPA (from top to bottom). 


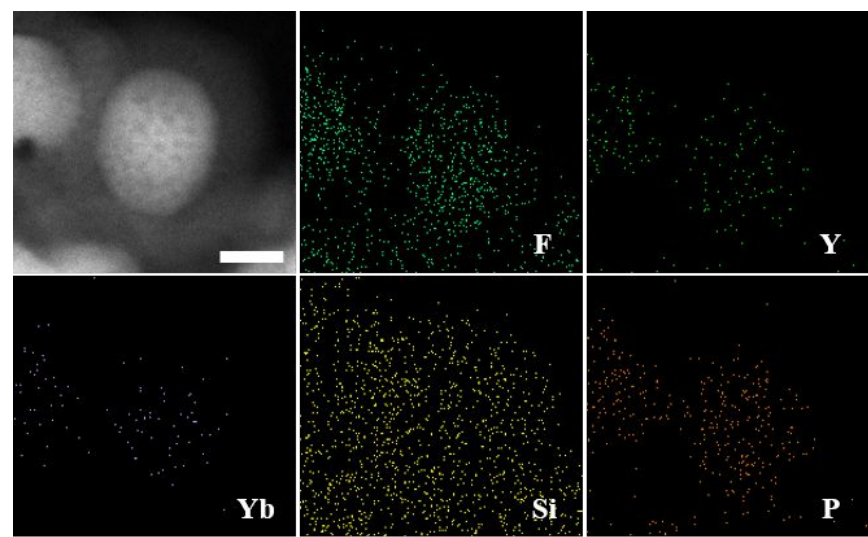

Figure S5. Elemental (F, Y, Yb, Si, P) mapping images corresponding to HAADF-STEM image of UCPA. Scale bar: $20 \mathrm{~nm}$.

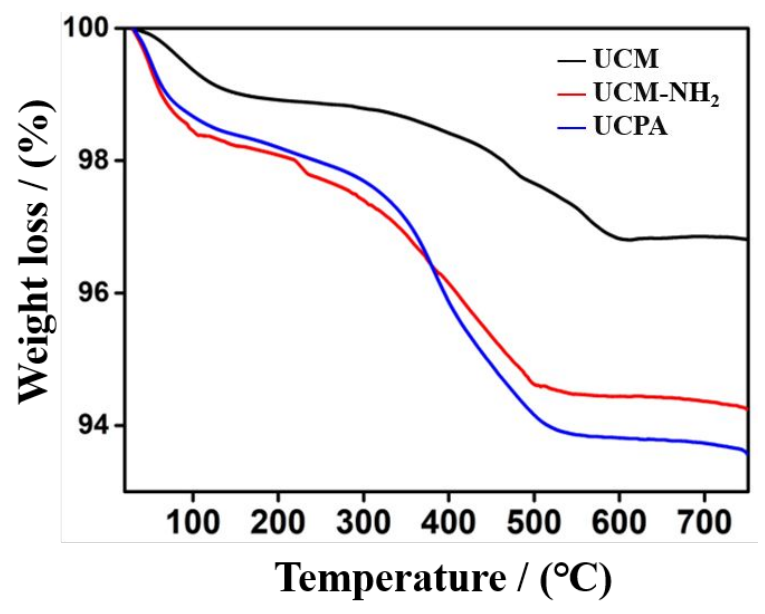

Figure S6. TGA curves of UCM, UCM-NH $\mathrm{N}_{2}$ and UCPA (from top to bottom). 


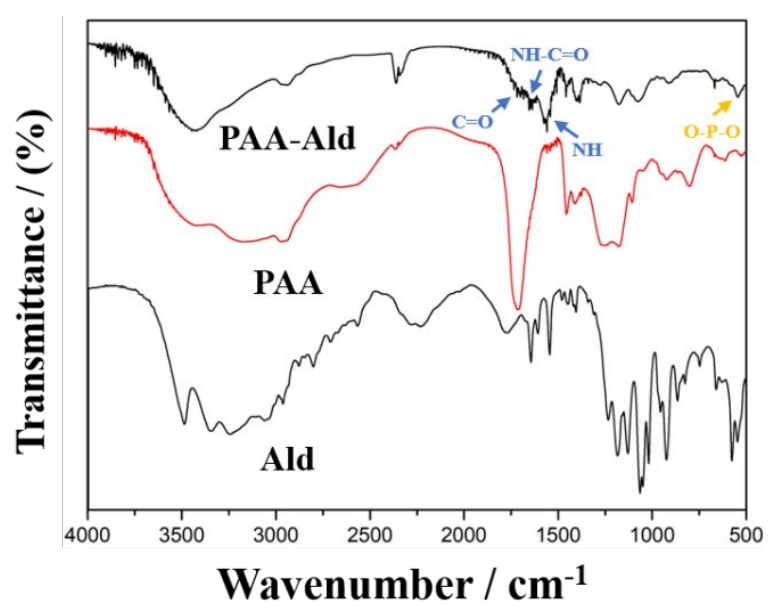

Figure S7. FT-IR spectra of Ald, PAA and PAA-Ald (from bottom to top).

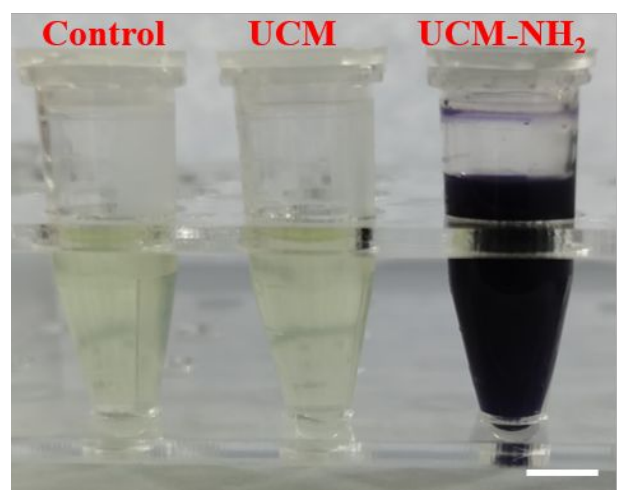

Figure S8. The results of the ninhydrin reaction of UCM and UCM-NH$H_{2}$. Scale bar: $1 \mathrm{~cm}$. 


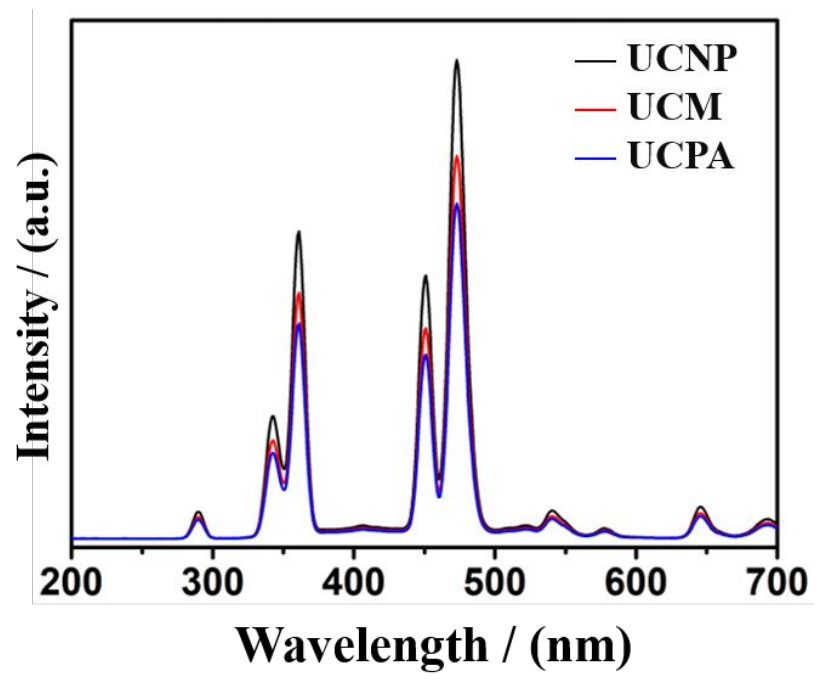

Figure S9. UCL spectra of UCNP, UCM and UCPA (from top to bottom).

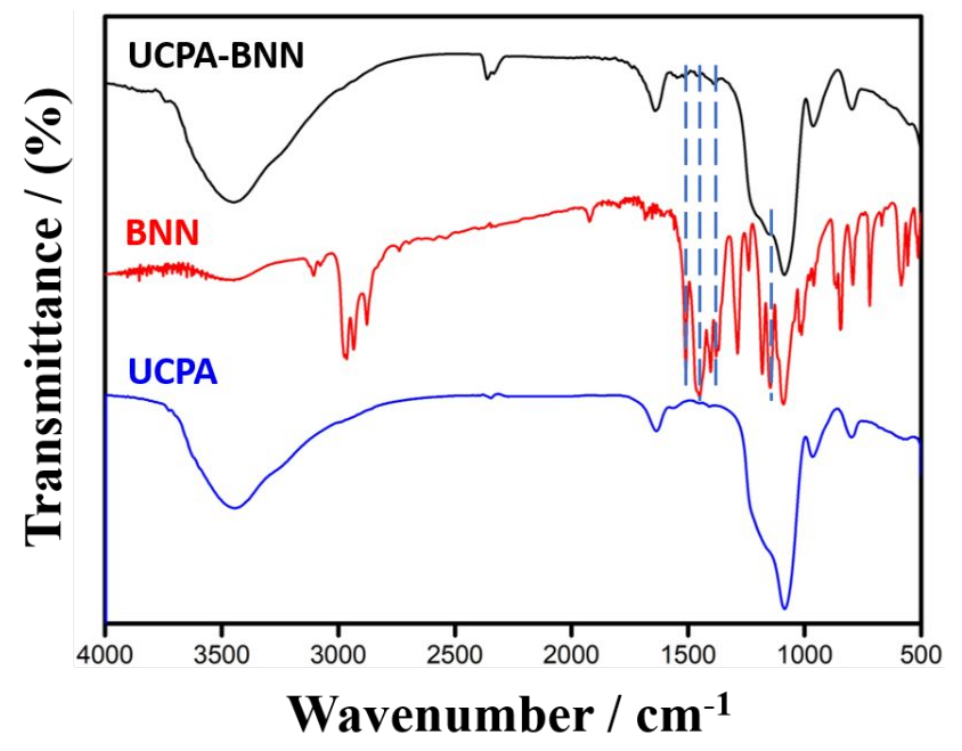

Figure S10. FT-IR spectra of UCPA-BNN, BNN and UCPA (from top to bottom). The dotted line represents the characteristic peaks of BNN appearing on the drug loaded UCPA (UCPA-BNN), indicating the successful loading of BNN in UCPA. 

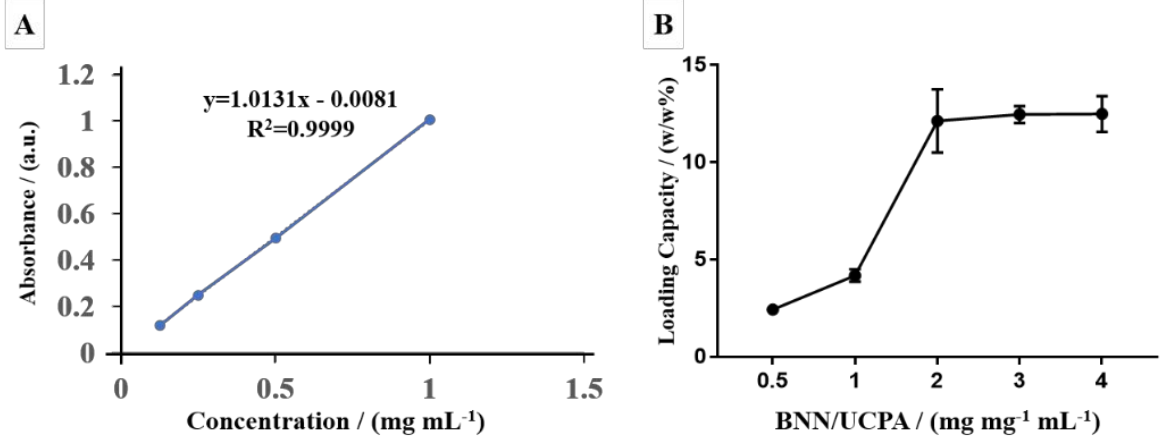

Figure S11. A) The standard curve of BNN in ethanol; B) The drug loading capacity of UCPA at different weight ratios of BNN/UCPA. Data are means \pm s.d. $(n=3)$.

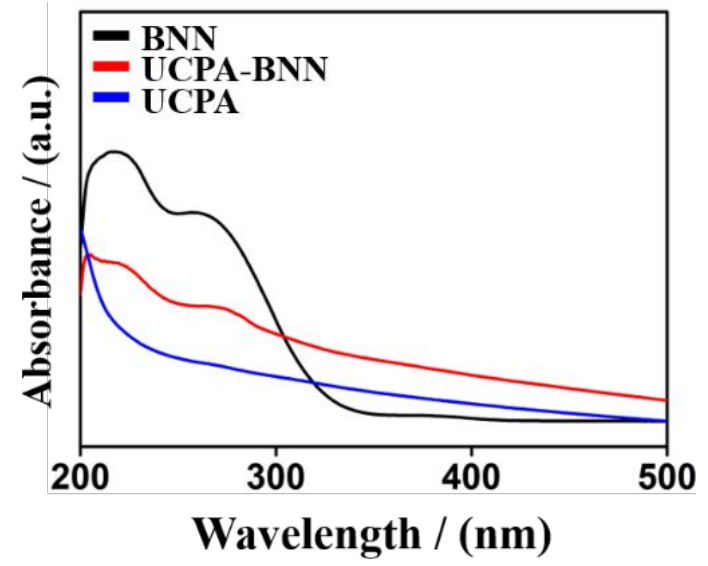

Figure S12. UV-vis absorption spectra of free BNN, UCPA-BNN and UCPA solutions (from top to bottom). 


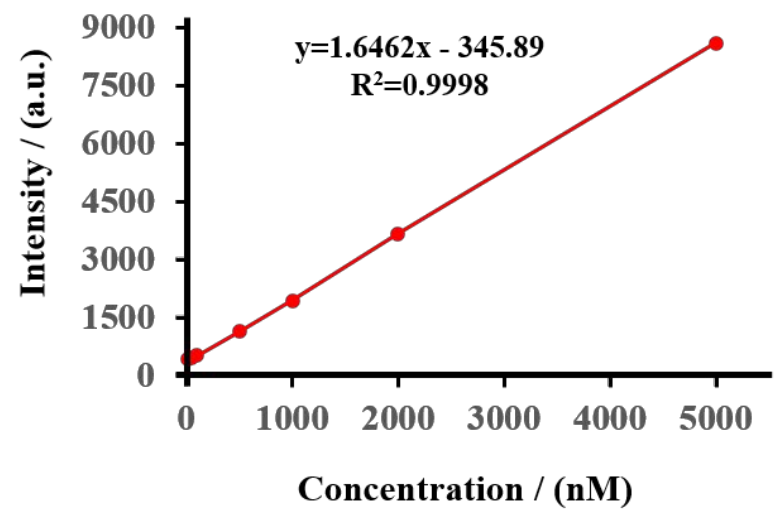

Figure S13. Standard work curve of $\mathrm{NO}$ measured by DAN $\left(\mathrm{NaNO}_{2}\right.$ as standard sample).

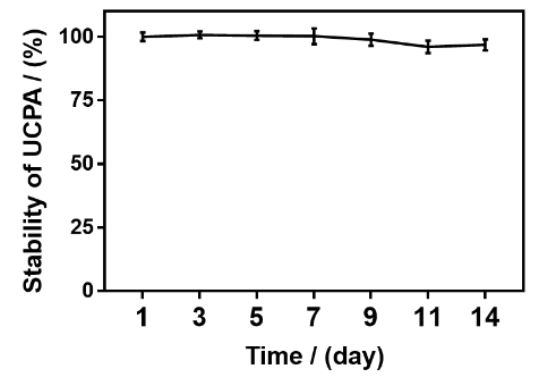

Figure S14. Stability of UCPA in 2 weeks in deionized water. These results showed their excellent long-term colloidal stability. Data are means \pm s.d. $(n=3)$. 


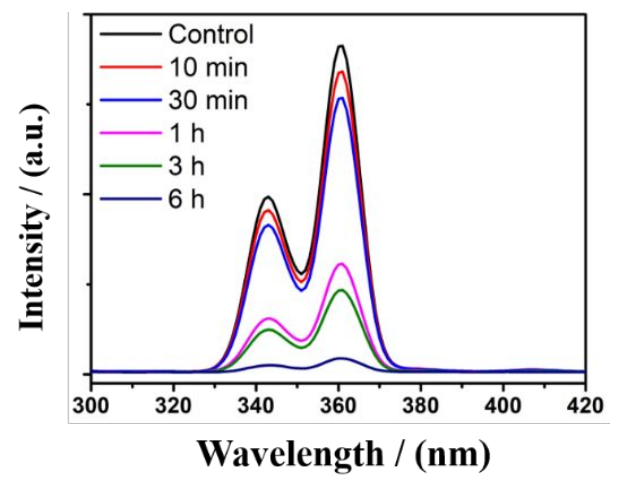

Figure S15. UCL spectra of supernatant of UCPA solution after stirring with HAP for different time.
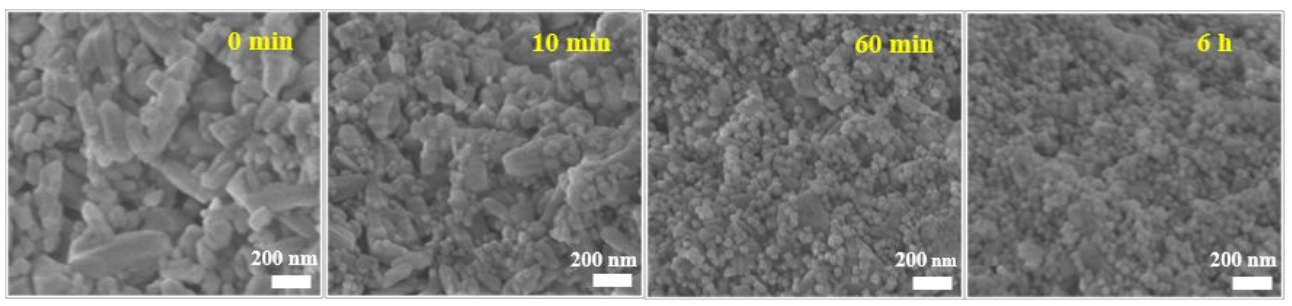

Figure S16. Representative SEM images of UCPA nanoparticles surface interactions with HAP after different time intervals as referred to Figure 3B.
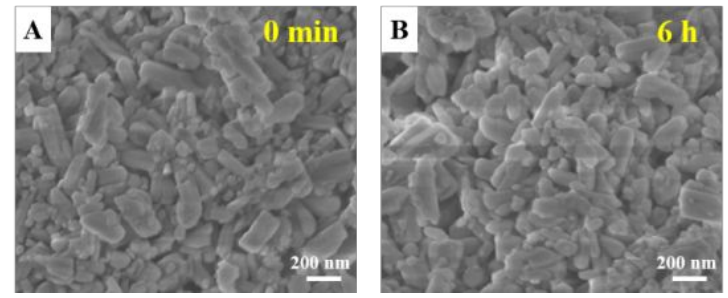

Figure S17. Representative SEM images of UCP nanoparticles surface interactions with HAP micro particle at $0 \mathrm{~min}$ and $6 \mathrm{~h}$. 


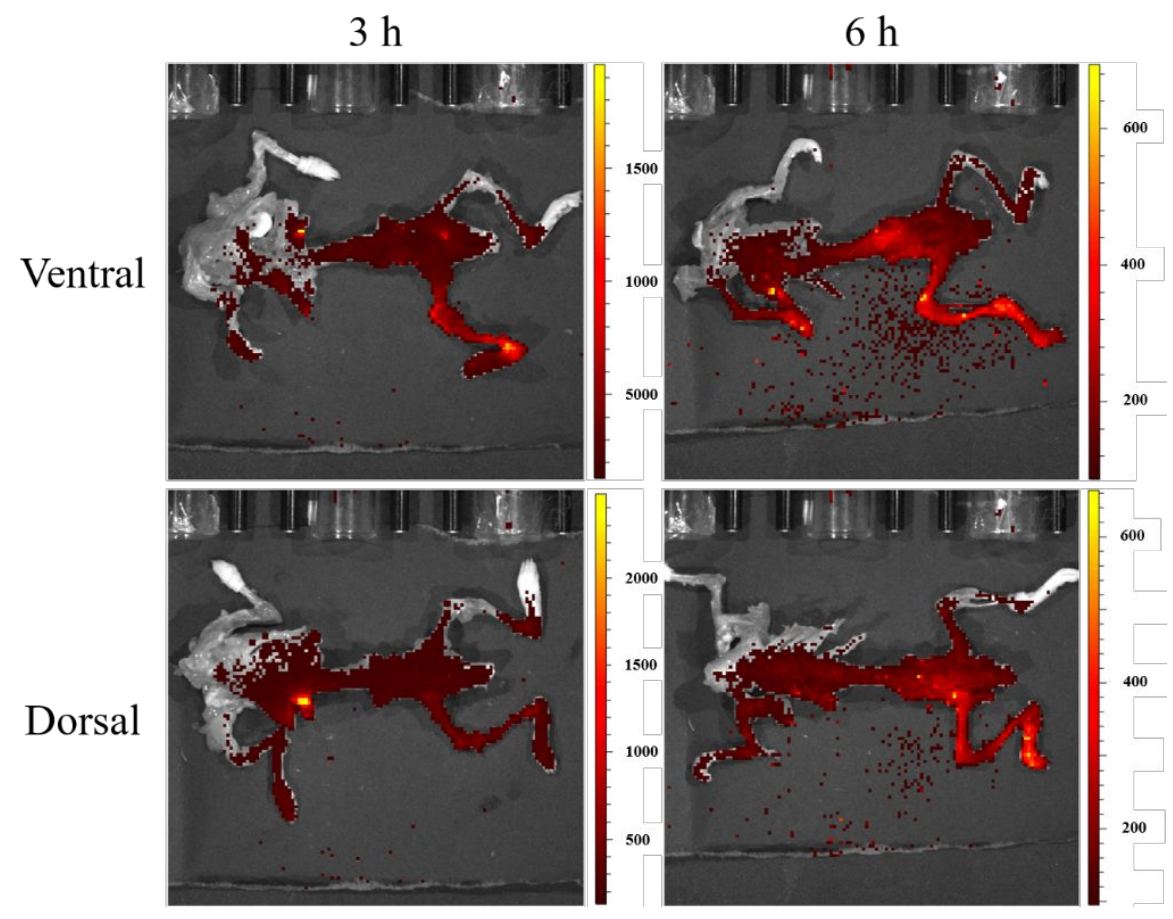

Figure S18. The in vivo fluorescence imaging of mice at $3 \mathrm{~h}$ and $6 \mathrm{~h}$ after intraperitoneal injection of UCPA.

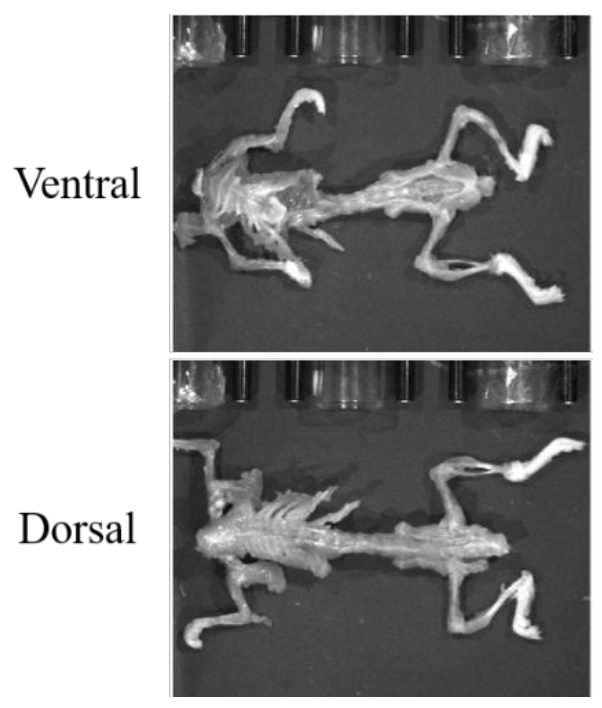

Figure S19. The in vivo fluorescence imaging of mice after $6 \mathrm{~h}$ of intraperitoneal injection of UCP. 


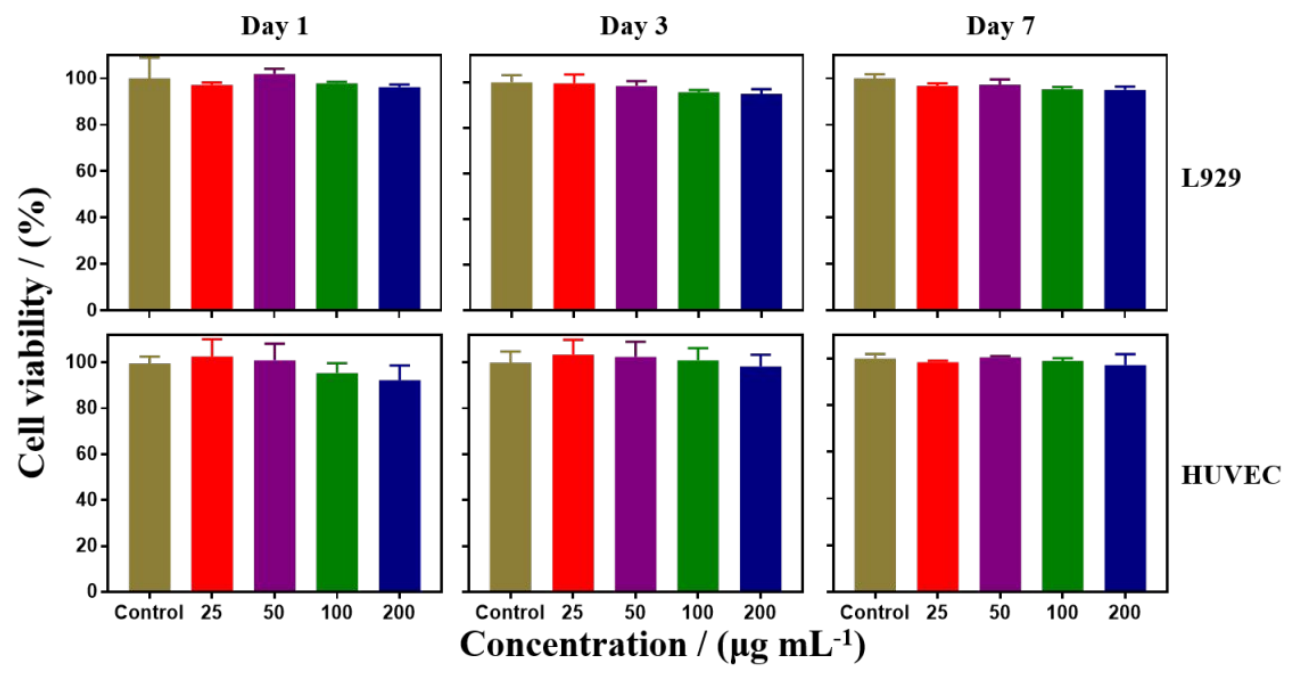

Figure S20. Cytotoxicity of UCPA-BNN at different concentrations to L929 and HUVECs cell lines at 1, 3 and 7 days, respectively. Data are means \pm s.d. $(n=3)$.

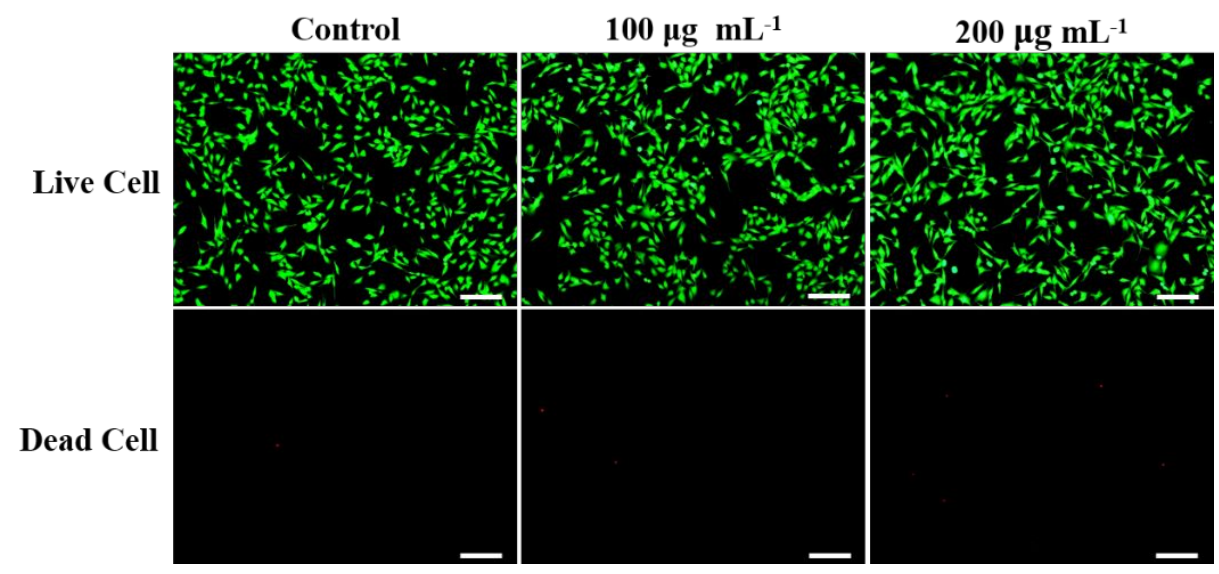

Figure S21. Live/Dead cell staining of MC3T3-E1 cells after coculture with different concentration of UCPA-BNN. Scale bar: $100 \mu \mathrm{m}$. 


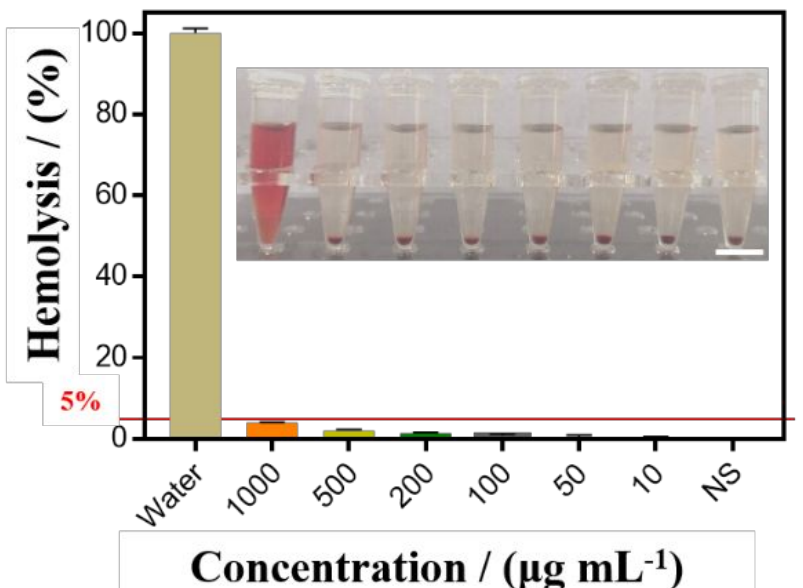

Figure S22. Hemolysis rate of UCPA-BNN at different concentrations. Data are means \pm s.d. (n

$=3)$. Scale bar: $1 \mathrm{~cm}$.
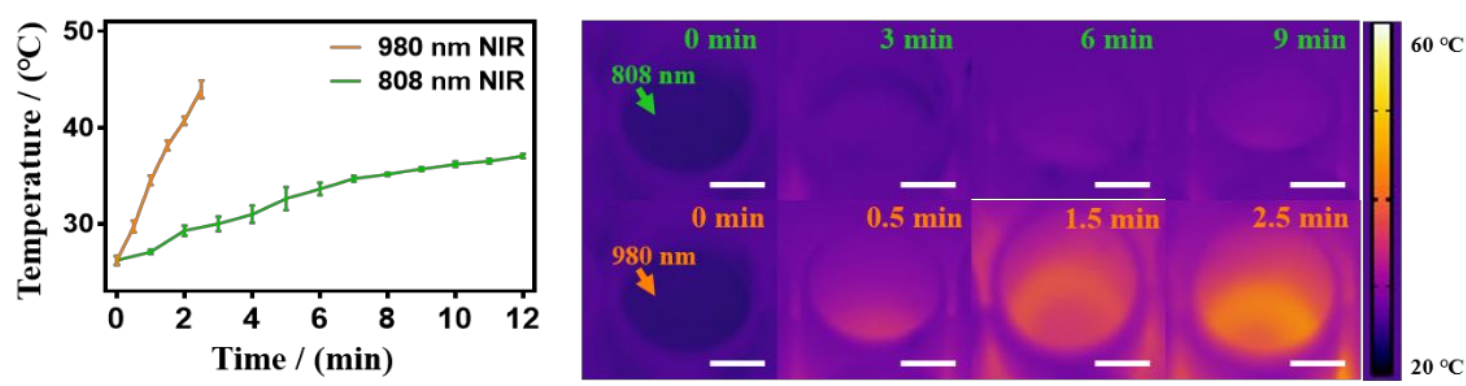

Figure S23. The heating curves and thermal imaging real photographs of ultrapure water irradiated by $980 \mathrm{~nm}$ and $808 \mathrm{~nm}$ laser, respectively. Data are means \pm s.d. $(\mathrm{n}=3)$. Scale bar: $1 \mathrm{~cm}$. 


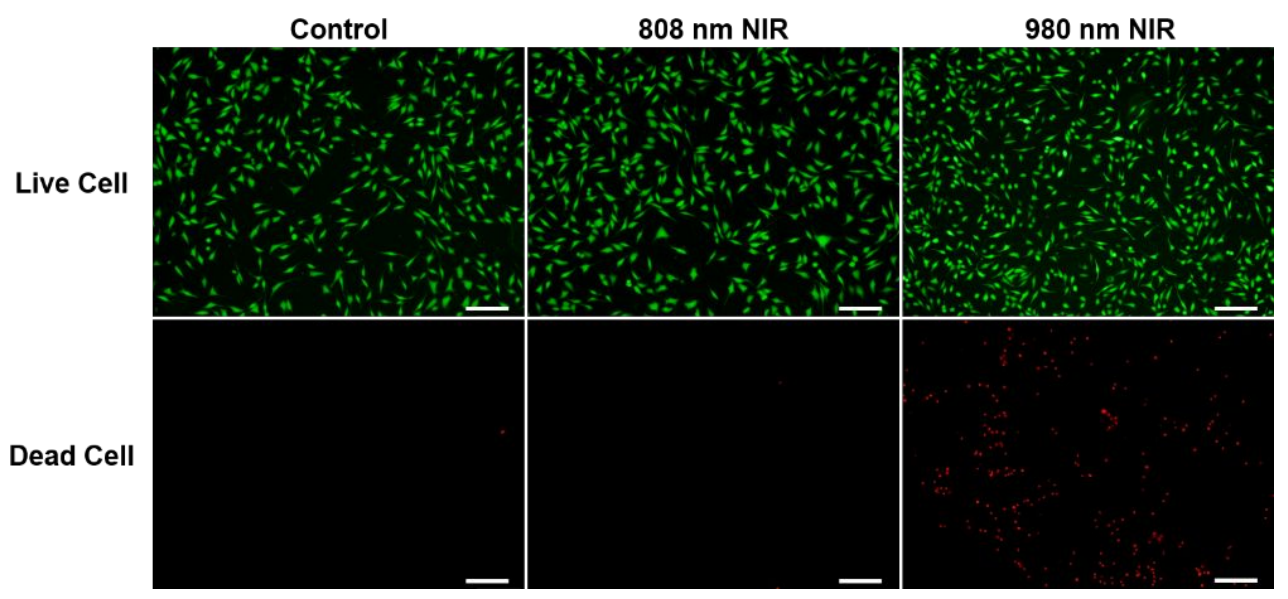

Figure S24. Confocal microscopy images of L929 cell irradiated by $980 \mathrm{~nm}$ and $808 \mathrm{~nm}$ laser, respectively. Scale bar: $100 \mu \mathrm{m}$.
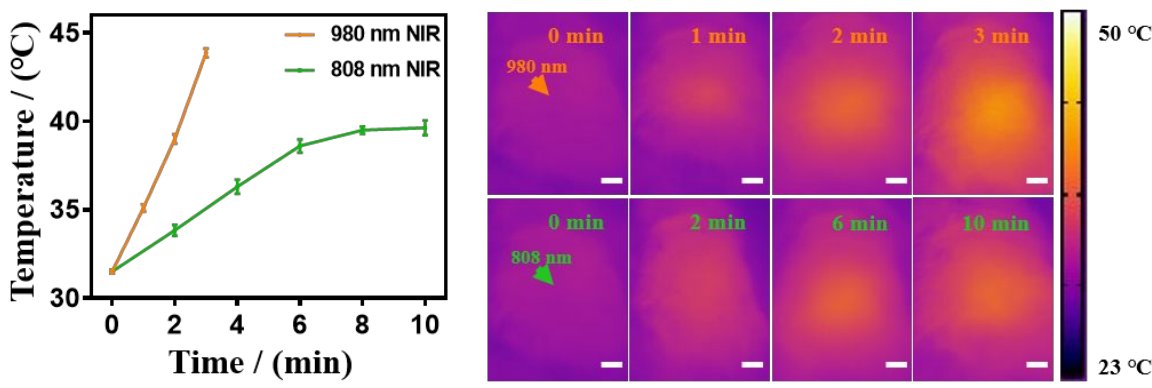

Figure S25. A) Irradiation time dependent temperature change. B-C) Digital photographs of a mouse upon 808 and $980 \mathrm{~nm}$ laser irradiation with the same power density. Data are means \pm s.d. $(\mathrm{n}=3)$. Scale bar: $1 \mathrm{~cm}$ 


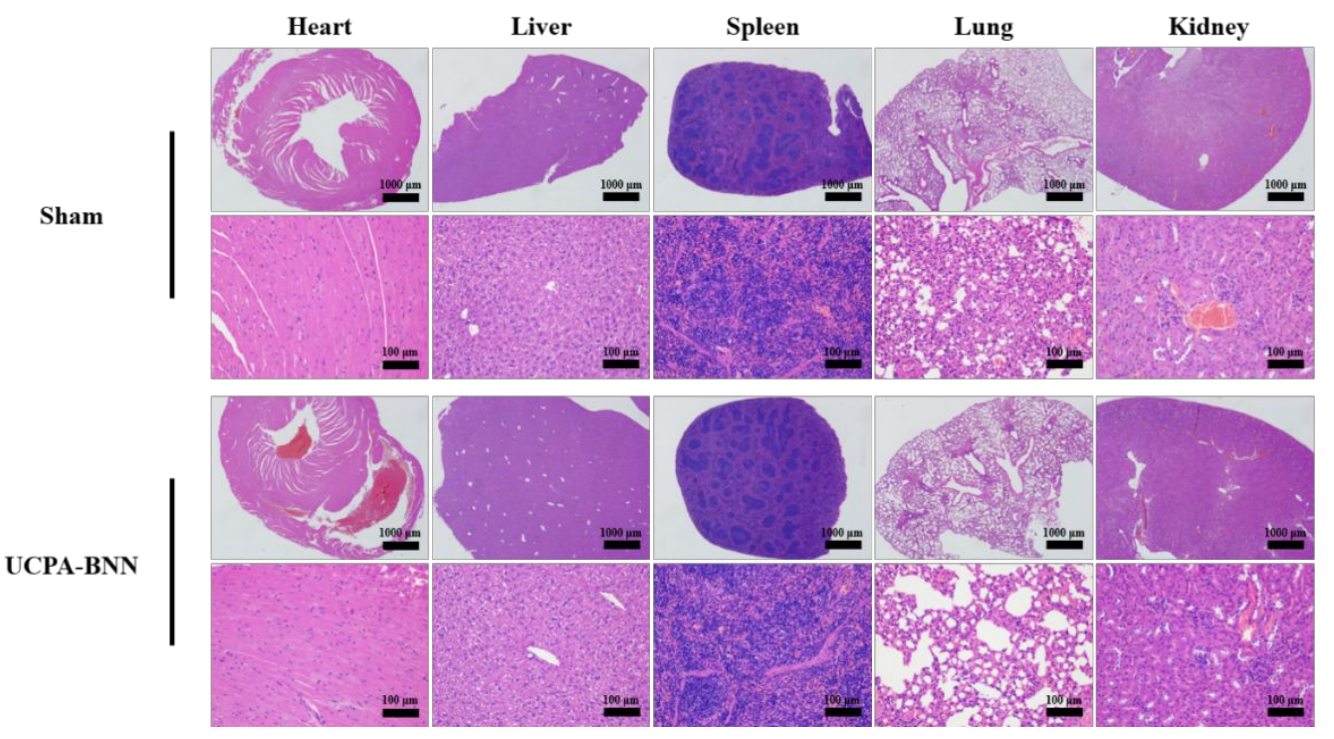

Figure S26. H\&E staining images of major tissues after 1 month of treatment in Sham group and UCPA-BNN group.

\begin{tabular}{|c|c|c|}
\hline Name & Forward primer & Reverse primer \\
\hline GAPDH & CCTCGTCCCGTAGACAAAATG & TGAGGTCAATGAAGGGGTCGT \\
\hline Col-1 & AAGAAGCACGTCTGGTTTGGAG & GGTCCATGTAGGCTACGCTGTT \\
\hline Runx2 & AGCGGACGAGGCAAGAGTTT & AGGCGGGACACCTACTCTCATA \\
\hline ALP & TGAGCGACACGGACAAGAAG & CCTGGTAGTTGTTGTGAGCGTAAT \\
\hline
\end{tabular}

Table S1. Primer sequence $\left(5^{\prime}-3^{\prime}\right)$ for the quantitative RT-PCR. 